\title{
Les organisations de producteurs au cœur de la valorisation de la qualité des fruits. Une diversité de stratégies en Rhône-Alpes
}

The strategic role of producers' organisations in improving the market recognition of quality in peach production. A diversity of strategies in the Rhône-Alpes region.

Sophie Dubuisson-Quellier, Mireille Navarrete et Jean Pluvinage

\section{Q OpenEdition}

\section{Édition électronique}

URL : http://journals.openedition.org/economierurale/715

DOI : $10.4000 /$ economierurale. 715

ISSN : 2105-2581

Éditeur

Société Française d'Économie Rurale (SFER)

Édition imprimée

Date de publication : 2 mai 2006

Pagination : 18-34

ISSN : 0013-0559

\section{Référence électronique}

Sophie Dubuisson-Quellier, Mireille Navarrete et Jean Pluvinage, « Les organisations de producteurs au cœur de la valorisation de la qualité des fruits. Une diversité de stratégies en Rhône-Alpes », Économie rurale [En ligne], 292 | Mars-avril 2006, mis en ligne le 05 mai 2008, consulté le 01 mai 2019. URL : http://journals.openedition.org/economierurale/715; DOI : 10.4000/economierurale.715 


\section{Les organisations de producteurs au cœur de la valorisation de la qualité des fruits. Une diversité de stratégies en Rhône-Alpes}

SOphie DUBUISSON-QUELLIER - Centre de sociologie des organisations-CNRS/Sciences Po, Paris Mireille NAVARRETE • INRA, Unité d'Écodéveloppement SAD, Avignon Jean PLUVINAGE •INRA-SAD, UMR Innovation, Montpellier

\section{Introduction}

L a filière fruits et légumes subit régulièrement des crises économiques depuis une dizaine d'années. Les consommateurs critiquent les prix trop élevés et l'insipidité croissante des fruits. Les producteurs sont toujours en situation limite de viabilité économique, ayant l'impression de faire les frais des négociations sur l'élargissement économique des marchés vers les pays du Sud. Les revenus des exploitations fruitières (par actif, en valeur réelle) augmentent plus faiblement que celles des autres secteurs (+ $1 \%$ par an, au lieu de $2 \%$ pour l'ensemble des exploitations, de 1991 à 2003) ${ }^{1}$.

L'action économique publique n'est pas facile dans un secteur marqué par une très grande diversité de produits périssables et saisonniers. Jusqu'en 1996, l'intervention publique était centrée sur le retrait du marché de certaines quantités de fruits les années de surproduction et l'octroi de compensations financières aux producteurs. $\mathrm{Ce}$ dispositif a entraîné des effets pervers, certains producteurs ayant adopté des pratiques de production conduisant vers une faible qualité, en se basant sur les prix de retrait.

\section{Un secteur en crise qu'il fallait réorganiser}

La réforme de 1996 de l'Organisation commune des marchés (OCM) a eu explicitement

1. Source: Agreste, Comptes par catégorie d'exploitations, Graphagri, 2005. pour objectif de réformer l'intervention publique, en la conditionnant à un effort important d'organisation économique et d'amélioration qualitative de la production $^{2}$. Les possibilités de retrait se sont drastiquement réduites ${ }^{3}$. Pour bénéficier des subventions européennes, les producteurs doivent désormais se constituer en Organisations de producteurs (OP) soumises à agrément, chargées de regrouper l'offre, de la mettre en marché et d'encourager des pratiques respectueuses de l'environnement. Chaque OP choisit parmi une liste d'actions éligibles (amélioration de la qualité au verger ou post-récolte, préservation de l'environnement, traçabilité des fruits...) celles sur lesquelles elle s'engage sur une durée de 3 à 5 ans, dans un « programme opérationnel » contractualisé et subventionné par l'UE. Ces financements sont plafonnés à 4,1\% de la valeur de la production commercialisée par l'OP, et le montant des subventions européennes est très faible comparativement aux autres secteurs de la production agricole ${ }^{4}$. La commercialisation par les OP, si elle est

2. Règlement CE 2200/96.

3. Entre 1996 et 2003, les taux nominaux de remboursement des pêches amenées au retrait sont passés de 20 à $11 €$ par quintal et le plafond de retrait de $50 \%$ à $10 \%$ du volume produit.

4. Par exploitation fruitière : $9300 €$ contre $37100 €$ pour les exploitations de grande culture et $28800 €$ pour les exploitations spécialisées en production de viande bovine (Agreste Rica, 2001). 
majoritaire en Rhône-Alpes ${ }^{5}$, laisse de côté une part de marché inorganisé, auquel sont attachés certains producteurs dans une période de crise du secteur et d'interrogation sur la capacité de régulation des pouvoirs publics. Ces changements sont cependant porteurs d'une évolution radicale, car il s'agit de passer du financement de la destruction de surplus à celui de l'amélioration des pratiques.

\section{La qualité comme résultat de la coordination par les OP}

Par cette réforme, les OP sont amenées à jouer un rôle central dans la qualification de la production et des produits, tant en amont dans le pilotage de la qualité des fruits qu'en aval dans sa valorisation par des stratégies de commercialisation adéquates. L'objectif de l'article est d' analyser cet acteur central de la coordination économique (Montigaud et al. 2002 ; Pluvinage, 2004) et son rôle dans la construction de la qualité des fruits. Plus précisément, nous montrons que ces qualités ne sont pas simplement le produit des exigences qualitatives exprimées par un aval plus ou moins univoque, mais qu'elles résultent de processus de coordination de l'OP entre les contraintes des producteurs en amont et les opportunités des clients en aval.

En observant comment ces structures cherchent à réguler des systèmes d'acteurs (Friedberg, 1993) de la production au marché autour de la qualité, nous adoptons une approche de la qualité qui rompt avec la démarche néo-institutionnaliste (Akerlof, 1970). D'une part, nous ne considérons pas que la qualité préexiste aux situations d'échanges, ce qui placerait les acteurs en position de la révéler ; nous considérons plutôt qu'elle se construit chemin faisant dans les négociations entre

5. En $200577 \%$ pour les pommes, $74 \%$ pour l'ensemble pêches-nectarines, $68 \%$ pour l'abricot, $45 \%$ pour la cerise. Source : chiffres du comité économique agricole des fruits et légumes Bassin Rhône Méditerranée (BRM). les différents acteurs, notamment entre l'OP, ses clients et ses apporteurs. D'autre part, fortement inspirés par l'économie des conventions (Eymard-Duvernay, 1989) et l'économie de la qualité (Karpik, 1989), nous nous intéressons aux conditions de production des accords locaux qui permettent aux acteurs de définir ensemble la qualité à partir des moyens dont ils se dotent pour l'évaluer (DubuissonQuellier, 2003a ; de Sainte Marie et Agostini, 2003). Nous rejoignons en ce sens les travaux sur la construction sociale de la qualité (de Sainte Marie et Valceschini, 1994) que nous complétons par une approche des relations marchandes (Cochoy et DubuissonQuellier, 2000). La qualité apparaît alors moins comme une propriété intrinsèque des objets que comme un produit de la relation d'échange (Dubuisson-Quellier, 2003b).

La qualité est au cœur des relations entre l'OP et les producteurs. Elle est produite par des individus sur leur exploitation agricole et organisée par l'OP ; mais c'est l'ensemble de son élaboration qui conditionne l'efficacité économique globale. Ainsi, Le Bail (2005) montre en quoi le bassin d'approvisionnement n'est pas seulement la somme de parcelles et de stratégies individuelles, mais aussi un espace de négociation entre l'OP et ses apporteurs. Plus particulièrement dans le secteur des fruits et légumes non stockables, c'est la coordination des producteurs sur des choix techniques (dates de plantation, choix de variétés) à l'échelle du bassin d'approvisionnement qui permet à l'OP d'échelonner suffisamment les dates de récolte pour atteindre le segment des Grandes et moyennes surfaces (GMS) (Navarrete et $a l ., 2003)$. En raisonnant la place des différents producteurs au niveau du collectif, il est possible de dégager de nouvelles marges de manœuvre, qui permettent d'atteindre les objectifs de qualité du collectif plus simplement ou à moindre coût que des solutions techniques individuelles (Navarrete et al., 2006). 


\section{Un dispositif de recherche interdisciplinaire}

Pour comprendre comment les OP pilotent l'élaboration de la qualité et construisent sa valorisation, nous avons étudié les stratégies de plusieurs OP localisées en RhôneAlpes $^{6}$ (Pluvinage et al., 2005). Cette région est à la fois le berceau originel de la production de pêche française, mais aussi le lieu emblématique de la crise qui secoue le secteur de la pêche depuis plusieurs années. En effet, il s'agit de la partie la plus septentrionale du bassin de production européen et sa production souffre de la concurrence de zones plus méridionales (Italie, Espagne, sud de la France) dont les productions répondent mieux aux standards actuels (précocité, teneur en sucre). Les années 2003 et 2004 traduisent des résultats économiques très décevants pour les producteurs ${ }^{7}$. Pourtant, tandis que certaines OP disparaissent ou sont en grande difficulté, d'autres se maintiennent. Sans nier l'importance de ces variables macroéconomiques, nous voulons montrer ici que les sources d'efficacité peuvent relever de différents modèles d'organisation et qu'il n'existe pas de modèle unique et optimal d'OP.

Pour étudier la capacité de coordination des OP, nous avons mis en place un dispositif méthodologique qualitatif spécifique, croisant regards sociologique et agronomique. Une enquête sociologique quasi

6. Cette étude a été financée dans le cadre d'un projet de recherche INRA PSDR sur la région Rhône-Alpes de 2002 à 2004 animé par J. Pluvinage et C. de Sainte Marie.

7. Globalement les prix des pêches et abricots en 2004, année de «petite récolte », sont inférieurs de $25 \%$ à ceux de la moyenne des cinq dernières années, incluant les valeurs de 2003 année de très fort gel en France et de prix $40 \%$ plus élevés que l'année précédente (source : le service des nouvelles du marché du ministère de l'Agriculture. exhaustive auprès des dirigeants des $17 \mathrm{OP}$ commercialisant la pêche en Rhône-Alpes (Puypalat, 2002) a été réalisée (ce qui représente plus de $80 \%$ du tonnage organisé), pour mettre au jour les principaux éléments sur lesquels les structures organisationnelles pouvaient varier. Nous avons alors formulé une grille d'analyse permettant de décrire les formes de commercialisation des produits et de coordination avec les producteurs dans toute leur diversité régionale ( $c f$. chapitre 2 , «De l'enquête sociologique à une grille d'analyse des OP »). À partir de ces résultats, nous avons identifié trois ensembles d'OP se différenciant selon les modalités concrètes d'organisation et de coordination avec les producteurs et avec les clients. Nous avons alors conduit des enquêtes monographiques très détaillées dans trois $\mathrm{OP}$, chacune représentative de l'un de ces trois modèles dans le but de décrire très finement les mécanismes de coordination entre les choix de production et les choix de mise en marché, à l'œuvre au sein de ce modèle d'OP. Ces enquêtes monographiques ont privilégié trois entrées : la fonction commerciale de l'OP, ses modes de coordination avec les producteurs et les modes de production des exploitations. Les monographies nous ont alors permis de construire trois idéaux-types d'OP que nous restituons dans cet article ( $c f$. chapitre 3, «Trois modèles typiques d'OP $\gg)^{8}$.

8. Dans cet article, ce sont les modèles que nous présentons et non les OP qui ont servi de terrain au travail monographique, cependant lorsque c'est nécessaire et pour rendre le propos plus clair, nous recourrons parfois à la présentation d'une réalité spécifique de l'une des OP enquêtées. 


\section{De l'enquête sociologique à une grille d'analyse des OP}

L'enquête sociologique conduite auprès des 17 OP de Rhône-Alpes souligne que cellesci ont de multiples façons de mettre en relation des modes de production, des qualités produites et des qualités commercialisables. Nous présenterons successivement la diversité des relations des OP avec l'aval et l'amont, en soulignant les spécificités de la production et de la mise en marché des pêches par rapport à d'autres productions. Il ne s'agit pas ici d'évaluer la compétitivité de la filière pêche en Rhône-Alpes, démarche qui nécessiterait une mise en perspective avec d'autres régions en France et en Europe, mais plutôt de comprendre ce qui explique que face à une situation partagée par les différents acteurs de la filière pêche (producteurs et metteurs en marché), différentes stratégies sont envisagées et mises en œuvre. Dans cette partie, nous rendons compte de cette situation commune aux différents acteurs.

\section{La relation avec l'aval : les contraintes et les opportunités de la mise en marché}

Les opérateurs de la filière, qu'il s'agisse des producteurs ou des metteurs en marché, soulignent le poids de la grande distribution sur le marché, ainsi que sa capacité à imposer un rapport de force très défavorable aux acteurs de l'amont. Chacun de ces acteurs sait qu'environ $70 \%$ des ventes de fruits et légumes se font dans les GMS (Hassan et Simioni, 2001), laissant la part congrue aux autres circuits de distribution. Ils décrivent souvent la grande distribution comme un macro-acteur qui ne traite que des produits standards laissant croire que les pratiques d'enseignes sont peu différenciées et reposent sur une segmentation de l'offre assez basique, fondée sur quelques critères (calibre, coloration, maturité, variété, et plus récemment le taux de sucre) dont les centrales savent instrumenter et contrôler la mesure. L'un des enjeux pour les enseignes apparaît lié à la construction de marques propres censées permettre de maîtriser la variabilité des apports imputable à l'atomisation de l'offre (Codron et al., 2003).

Il est vrai que cette recherche de la régularité est importante pour les enseignes qui veulent ainsi préserver l'existence d'un rayon fruits et légumes dans leurs magasins, rayon qui contribue à fidéliser les consommateurs. Les démarches de segmentations stratégiques pratiquées par les différentes enseignes dans le secteur des fruits et légumes (Simon, 2003) sont encore très rudimentaires. Les magasins ne distribuent au mieux leur offre que sur trois segments :

- un premier prix,

- un cœur de marché correspondant à une qualité dite « standard » qui est une sorte de contrat minimal autour du produit,

- une qualité différenciée par l'enseigne (appelée «standard + »).

Malgré tout, sur la base de cette segmentation simple, les travaux de Simon montrent que les magasins font tout de même varier les critères (variétaux, d'emballage, de label, de calibres) rendant l'offre assez peu lisible pour le consommateur. Par exemple, dans la catégorie des produits de qualité « standard + », on trouve à la fois des pêches standards en vrac de calibre A ou B, du label $\mathrm{AB}$ ou encore des barquettes de pêches mûres à point. Enfin, les écarts de prix peuvent aller du simple au double entre produits premiers prix et produits de qualité standard ou du simple au quadruple avec des produits de qualité différenciée. Une telle hétérogénéité observée représente un contrepoint troublant aux discours des producteurs qui mettent plutôt en avant l'homogénéisation de l'offre produite par la distribution de masse.

En réalité, il faut probablement distinguer deux aspects : d'une part une tendance de la grande distribution à chercher des approvisionnements stables et réguliers, d'autre part sa volonté, dans certaines circonstances de 
traiter une sorte de forme d'hétérogénéité de l'offre dans des stratégies de différenciation contrôlées. Autrement dit, la grande distribution cherche avant tout à limiter l'expression d'une trop forte variabilité parmi les produits qu'elle achète pour, au contraire, envisager des modes de valorisation autour de produits spécifiques dont elle aura elle-même travaillé la différenciation.

Un autre élément essentiel dans la relation de l'OP avec l'aval est sa capacité à capter très tôt les marchés au démarrage de la campagne de production. En effet, la grande distribution absorbe des volumes conséquents qu'elle négocie contre des prix très tendus (Barrey, 2004). Pour les metteurs en marché, et spécifiquement ceux qui concentrent des tonnages importants d'un produit par nature peu stockable et fragile, il devient donc tout à fait essentiel de capter cette demande. L'économie de la captation (Trompette, 2005) vise à mettre le client le plus rapidement possible dans une relation marchande dont il devient coûteux de s'extraire. Dans le cas des fruits d'été, la prime est donc donnée à l'OP qui peut mettre des produits sur le marché avant les autres. Un paradoxe se fait jour : plus les metteurs en marché s'organisent en se concentrant, pour faire face au pouvoir de la grande distribution, plus ils en sont objectivement dépendants : la grande distribution devenant ainsi le seul acteur à même d'écouler leurs volumes croissants, et plus la captation se pose dans les termes d'un accès anticipé au marché par la vente de produits précoces. Nous tenons ici les dimensions principales des stratégies qui poussent les metteurs en marchés rhône-alpins à se concentrer et à s'approvisionner en produits précoces, par l'achat de pêches ou l'acquisition de vergers plus au Sud (Bouches-du-Rhône, Gard, voire Espagne).

Mais la grande distribution ne constitue pas la totalité des débouchés possibles pour les produits. Un tiers des ventes passe par les circuits traditionnels (marché de gros ali- mentant le commerce de détail, la restauration, les revendeurs forains, et parfois aussi indirectement la grande distribution). Ces circuits sont souvent marqués par des relations de longue date construites entre ces grossistes et des producteurs ou des OP. Ils reposent sur la constitution de lots de produits de volume plus limité, avec des positionnements qualitatifs variables, mais souvent plus orientés vers la qualité supérieure et une plus grande fraîcheur. Enfin, il faut ajouter les débouchés vers la transformation pouvant représenter $10 \%$ des ventes pour certains metteurs en marché. Mais aujourd'hui, ce débouché peu rentable vise principalement à écouler les produits non commercialisables sur le frais, permettant notamment de réintégrer une partie des fruits qui partaient auparavant au retrait.

En résumé, les relations avec l'aval sont très structurées par la place qu'y joue la grande distribution. Emergent ainsi deux formes un peu typiques de relations avec l'aval. L'une fortement orientée vers des stratégies de volume destinées à faire contre poids à la concentration de l'aval, l'autre au contraire visant la différenciation des qualités dans des circuits ad hoc.

\section{La relation avec l'amont : ressources et moyens de production}

Rechercher une adéquation entre qualités produites et qualités commercialisables est donc un enjeu pour toutes les OP. À partir de l'enquête exhaustive et de la littérature, nous identifions ici divers modes de coordination avec les producteurs qui permettent de piloter des composantes de la qualité. Nous nous focalisons sur la teneur en sucre, la précocité des récoltes et le degré d'hétérogénéité des produits, critères de qualité les plus discriminants entre les différents circuits de commercialisation. À partir des connaissances sur les déterminants agronomiques de la qualité, nous décrivons les possibilités qu'ont les OP de gérer ces critères de qualité, à trois pas de 
temps : pluriannuel, annuel et infra-annuel.

Les potentiels de teneur en sucre et de précocité dépendent de deux facteurs définis sur le long terme : la localisation des parcelles et le choix des variétés. En un lieu donné, le choix des variétés détermine à la fois la date d'entrée en production (entre mijuin et mi-septembre) et sa teneur en sucre (de $8^{\circ}$ Brix $^{9}$ pour les variétés acidulées à $14^{\circ}$ Brix pour les variétés douces) (Giauque et al., 1997). Mais du fait des différences d'ensoleillement et de pluviométrie entre Rhône-Alpes et les Bouches-du-Rhône ou le Gard, la date de récolte pour une même variété de pêche est décalée de 20 jours, la teneur en sucre augmentée de plusieurs degrés Brix (Vidal, 2003). Très en amont du processus de production d'un lot de pêche, l'OP peut donc sélectionner les exploitations qui l'approvisionnent sur la base de leur localisation géographique ou des caractéristiques variétales de leurs vergers, afin de faire converger au mieux les potentialités agronomiques des exploitations et la stratégie commerciale de l'OP. Elle peut également inciter les exploitations à faire évoluer leurs vergers par arrachage et replantation de variétés plus adaptées.

Une fois la plantation effectuée, la teneur en sucre des pêches dépend fortement des conditions climatiques et de l'itinéraire technique de l'année, principalement l'irrigation, l'éclaircissage des fruits, la taille en vert réalisée après la récolte (Aulagnier, 1996 ; Giauque et al., op. cit.). L'OP peut donc encourager des changements de pratiques culturales par un cahier des charges à la production. Cependant, malgré ces interventions techniques, il demeure, pour des raisons biologiques, une forte variabilité de qualité intra-arbre.

Enfin, la teneur en sucre est conditionnée par le choix de la date de récolte par rapport

9. Le degré Brix $\left({ }^{\circ} \mathrm{B}\right)$ indique la fraction de sucre dans un liquide, il permet de mesurer la maturité des fruits. à la date de maturité effective du fruit et par le nombre de récoltes par parcelle. L'OP peut donc tenter d'infléchir les conditions de récolte (date, nombre de passages), mais sa principale marge de manœuvre se situe en station de conditionnement, par le tri des produits récoltés. Les calibreuses électroniques capables de trier en ligne chaque fruit individuellement sur des critères variés (calibre, couleur, teneur en sucre) et qui équipent la plupart des $\mathrm{OP}$, redonnent potentiellement un rôle stratégique à cette phase. Cependant, s'agissant d'un fruit périssable comme la pêche, l'affectation d'un lot à un client ne peut être retardée plus de quelques jours, ce qui limite les marges de manœuvre des OP dans ce processus d'allotement.

L'hétérogénéité de la production peut être partiellement contrôlée au niveau de l'OP. Deux stratégies extrêmes peuvent être identifiées :

- la première consiste à attendre la même qualité de tous les producteurs quelles que soient les différences de structure et d'organisation des exploitations ou leurs conditions pédoclimatiques, dont on sait qu'elles sont pour partie responsables de l'hétérogénéité de qualité ;

- à l'opposé, une autre stratégie consiste pour l'OP à reconnaître que les exploitations ont des capacités différentielles à produire une qualité donnée, et à chercher à valoriser au mieux cette diversité. Par exemple, dans le premier cas, l'OP exige une teneur en sucre élevée indépendamment de la localisation des exploitations, tandis que dans le second elle attribue à chaque exploitation un segment de marché particulier en fonction de leurs propres potentialités.

\section{Construction d'une grille d'analyse des OP}

À partir de cette double description des conditions d'organisation des relations avec l'aval et l'amont, nous proposons une grille analytique pour penser cette diversité des modes d'organisation des OP (tableau 1). 
Tableau 1. La coordination de l'OP avec l'amont et l'aval

\begin{tabular}{c|l}
\hline $\begin{array}{c}\text { Critères pour décrire la coordination } \\
\text { avec l'amont }\end{array}$ & \multicolumn{1}{c}{$\begin{array}{c}\text { Critères pour décrire la coordination } \\
\text { avec l'aval }\end{array}$} \\
\hline - Période de coordination pour orienter les \\
$\begin{array}{c}\text { changements de pratiques (plantation, itinéraire } \\
\text { technique annuel, récolte) }\end{array}$ & $\begin{array}{l}\text { - Formes de la relation client } \\
\text { - Type de compétence marchande } \\
\text { - Modalités et degré de valorisation de la diversité } \\
\text { des exploitations du bassin d'approvisionnement }\end{array}$ \\
- Modalités et degré d'incitation & - Formes de valorisation de la diversité des qualités \\
\hline
\end{tabular}

Pour décrire la coordination avec la production, nous retenons trois critères :

- le premier décrit le degré d'anticipation des changements techniques dans les échanges entre l'OP et ses adhérents (long, moyen, court terme) ;

- le deuxième critère décrit les modalités et le degré de valorisation de la diversité des exploitations agricoles du bassin d'approvisionnement ;

- le troisième critère porte sur le degré d'incitation des OP au changement de pratiques.

Pour décrire les formes de commercialisation, nous retenons quatre critères:

- la relation client permet de suivre la façon dont l'OP envisage la demande, se donne des moyens de suivre ses variations et spécifie ses circuits aval ;

- le deuxième critère décrit la compétence marchande de l'OP à travers sa capacité à animer son marché et à s'y adapter ;

- le troisième critère porte sur la manière dont l'OP crée de la valeur et constitue des rentes de situation à partir des circuits commerciaux avec lesquels elle travaille ;

- enfin le dernier critère concerne la valorisation marchande de la diversité des qualités et décrit la manière dont l'OP tra- vaille une relation au marché qui lui permet de tirer partie de cette diversité.

\section{Trois modèles typiques d'OP}

À partir des critères d'analyse précédents, il est alors possible de tracer deux axes qui décrivent, pour l'un le degré de valorisation de la diversité des qualités par les circuits commerciaux et pour l'autre, le degré de coordination avec les producteurs. En croisant les positions des OP sur ces deux axes, nous faisons apparaître quatre idéaux-types de stratégies d'OP, dont trois sont présentés (tableau 2). $\mathrm{Si}$ nous souhaitons insister sur le fait que ces idéaux-types relèvent d'une modélisation qui tend à durcir certaines tendances et, en ce sens, ne décrit pas la réalité concrète de telle ou telle OP, nous précisons malgré tout que les éléments qui nous ont aidés à construire les critères de typologie relèvent quant à eux de situations réellement rencontrées dans nos enquêtes.

En conclusion, nous reviendrons sur le quatrième modèle, qui semble décrire une réalité moins prégnante ou peut-être en voie de régression dans le paysage actuel des OP du bassin économique du sud-est.

\begin{tabular}{|c|c|c|c|}
\hline \multirow{2}{*}{\multicolumn{2}{|c|}{ Tableau 2. Les modèles d'OP }} & \multicolumn{2}{|c|}{ Valorisation de la diversité des qualités } \\
\hline & & Faible & Forte \\
\hline \multirow[t]{2}{*}{ Coordination avec les producteurs } & Faible & $\begin{array}{c}\text { Coopérative } \\
\text { «traditionnelle » }\end{array}$ & Fédératif \\
\hline & Forte & Hiérarchique & Club \\
\hline
\end{tabular}


1. Le modèle hiérarchique : un écran relativement " opaque " entre des producteurs et des clients

Ce modèle se caractérise par la volonté de l'OP de constituer une structure organisationnelle forte destinée à coordonner de manière très centralisée les opérations de l'amont et celles de l'aval de sorte que producteurs et clients ne soient pas directement en contact.

\section{Une segmentation stratégique de la} demande sur un petit nombre de variables

La stratégie commerciale adoptée par les OP se référant à ce modèle est fondée sur le volume et la concentration de l'offre, comme réponse efficace à la concentration de la demande (grande distribution française et étrangère). Ce positionnement n'exclut donc pas les circuits traditionnels via les grossistes, à condition qu'ils soient de dimension nationale, voire internationale. Cette stratégie de volume impose un modèle d'organisation particulier qui vise à stabiliser la relation client de plusieurs manières : en développant des compétences commerciales pour proposer des interlocuteurs identifiables et pérennes aux clients, en contractualisant lorsque c'est possible les engagements de part et d'autres ${ }^{10}$ et en investissant durablement dans la relation par des stratégies de services associées (adaptation du conditionnement, dépannage de dernière minute, priorité accordée en cas de pénurie de l'offre). Dans cette configuration, le marché de la pêche est associé à un marché de la demande, puisque c'est elle qui est le signal déclencheur en matière de gestion de la production : orientation de la mise en production, déclenchement de la cueille, du

10. Cahiers des charges produit qui permettent d'objectiver certaines des caractéristiques de l'offre et de garantir sa stabilité dans le temps ; engagements oraux sur les volumes et les qualités attendues en fin de campagne pour la suivante ; engagements plus précis établis un à deux mois avant le démarrage des récoltes. transport jusqu'à la station de conditionnement et du conditionnement. S'agissant de produits élaborés sur un pas de temps annuel, voire pluriannuel (pour les variétés), cela suppose que l'OP connaisse l'offre longtemps à l'avance pour pouvoir répondre aux demandes qui lui sont formulées, et que la production ne varie pas de manière trop multidimensionnelle.

Mais il est clair aussi que symétriquement, ces opérations ne sont possibles que si l'on s'est également assuré que la demande reste prévisible. C'est la raison pour laquelle ce modèle repose sur une segmentation du marché volontairement très simple, articulée autour de critères très objectivables (taux de sucre, variété, maturité, calibre), et sur un petit nombre de segments de marché, sur laquelle les différents clients doivent pouvoir se distribuer. Ce modèle d'OP fonde donc sa rente de situation sur une forte prévisibilité de son positionnement marchand : les opérateurs de l'amont (producteurs) et de l'aval (clients) peuvent planifier très largement leurs opérations et compter sur une forte stabilité dans le temps de l'offre et de la demande. Mais, on le voit bien, cette stabilité des actions de l'OP n'est acquise qu'au prix d'une réduction très forte de la variabilité des produits qui y circulent. Le modèle ne tient plus si en amont les producteurs lui fournissent une grande diversité de qualité.

\section{Une coordination très poussée des producteurs}

Les OP s'orientant vers ce modèle ont par conséquent la volonté de peser fortement sur les pratiques des producteurs.

Une partie du conseil technique est contrôlée par l'OP elle-même, pour les techniques ayant un effet important sur les critères jugés cruciaux par l'OP (l'homogénéité des produits, les dates de récolte). C'est notamment le cas pour le choix des variétés : une première liste de variétés recommandées à la plantation (parmi les 400 variétés disponibles) est établie pour 
réduire la diversité variétale dans le bassin d'approvisionnement, donc la diversité des produits ; elle ne comporte qu'une variété par créneau de date (Rouby, 2001). Une deuxième liste regroupe les variétés à arracher car elles ne satisfont plus les exigences actuelles des clients. Une grille de prix établit le niveau des primes et des réfactions appliquées pour les variétés de chaque liste par rapport au prix de vente effectif aux clients. Ces listes sont élaborées par le technicien et le commercial et réactualisées chaque année en fonction des innovations variétales et des performances commerciales des variétés. Le technicien cherche également à coordonner les choix techniques des producteurs pour que les volumes de fruits produits collectivement soient étalés sur la plus longue période possible.

S'agissant en majorité d'OP anciennes et à la recherche de volumes (pour rentabiliser leurs infrastructures et maintenir leur poids dans la grande distribution), elles ne peuvent sélectionner trop sévèrement les producteurs qui souhaitent adhérer. Pour dépasser la forte diversité entre exploitations, il existe un système de notation très sévère des lots à l'agréage qui joue un rôle crucial dans le dispositif de pilotage de la qualité. L'agréeur trie les lots de pêches à l'entrée en station, sur des critères de qualité mesurés (taux de sucre, calibre...), mais aussi en fonction des potentialités globales des exploitations, afin d'orienter au mieux les lots en fonction des attentes des clients. Pour compenser ce processus, sur lequel les producteurs n'ont aucune prise, ils opèrent eux-mêmes un tri en parcelle et n'apportent à l'OP que ce qu' elle sait effectivement valoriser.

\section{La centralisation comme moyen d'ajustement entre production et marché}

Ce modèle est fondé sur une organisation très centralisée des opérations de l'OP. La coordination des producteurs d'un côté et les relations marchandes de l'autre font l'objet de procédures fortement stabilisées dans des dispositifs (contractuels ou organisationnels). Par exemple, un bilan post-saison, en fin d'année civile, permet à l'OP de restituer aux producteurs les performances commerciales de tel ou tel type de qualité (notamment variétale) ou sur une période donnée. Cette information permet à l'OP de justifier la programmation de réorientation des plantations, mais également la mise en place de mesures de rémunération incitatives pour que les producteurs adaptent leur production aux évolutions du marché. De la même manière, la procédure de prévision des récoltes un mois avant celles-ci permet à l'équipe commerciale de connaître assez précisément la manière dont l'offre va se répartir entre différentes qualités et dans le temps pour pouvoir faire des propositions commerciales avant même que les produits ne soient rentrés en station. Dans les relations entre producteurs et service commercial, qui peuvent devenir conflictuelles en raison de la hauteur des enjeux pour chaque partie, le technicien a un rôle d'interface crucial, cherchant un équilibre entre les intérêts commerciaux de l'OP et les capacités de production individuelles de chaque exploitation.

Ce modèle incite donc les OP à organiser les conditions d'un approvisionnement particulier : des forts volumes, garantis par le recours à des bassins de production importants et à une coordination technique forte cherchant à limiter la variabilité qualitative, un accès au marché le plus tôt possible obtenu par la délocalisation d'une partie de la production plus au sud, des approvisionnements complémentaires, hors bassin par l'intermédiaire d'une SA.

La segmentation de la demande, fondée sur un faible nombre de critères, permet à l'OP de coordonner la production. Les autres formes de variabilité sont en quelque sorte absorbées par l'organisation, qui s'arrange pour qu'elles ne s'expriment pas plus en aval. Ce modèle, qui ne tient qu'au prix 
d'une très forte centralisation des informations et coordination des partenaires, est assez proche de formes de gestion de la production industrielle en flux poussés.

\section{Le modèle fédératif : des circuits commerciaux adaptés à une valorisation de la qualité du produit}

Le modèle fédératif est le contrepoint du modèle hiérarchique, puisque la coordination avec les producteurs y est faible tandis que la valorisation de la diversité des qualités y est, au contraire, forte.

\section{Valoriser au mieux la diversité des qualités produites}

L'objectif de ce modèle d'OP est de valoriser les qualités des produits dans les circuits commerciaux les plus adaptés à celles-ci. La structure organisationnelle se veut donc légère et de nature fédérative. Les producteurs pilotent eux-mêmes les circuits de commercialisation (s'ils sont producteurs-expéditeurs) ou commercialisent par des expéditeurs avec lesquels des relations se sont établies de longue date, souvent dès les générations antérieures.

Contrairement au modèle précédent, ici la relation client est d'autant plus profitable qu'elle est flexible. Poussé à l'extrême, ce modèle peut même viser la mise en concurrence des circuits et donc des clients comme modalité de création de valeur. Car la vision du marché de la pêche dans une telle configuration est celle d'un marché de l'offre. Les producteurs mettent sur le marché des lots de qualité très diverses (petits ou gros calibres, fruits très aromatiques ou peu goûteux, sucrés ou non, ...), et cherchent à exploiter le plus grand nombre de facettes possibles du marché en s'adressent à des opérateurs diversifiés : expéditeurs, grossistes, enseignes décentralisées, chefs de rayon ou directeurs de magasins, exportateurs. Dans cette relation marchande, sont mis en avant non pas seulement des critères de qualité des lots mais également des critères caractérisant le producteur lui-même (sa réputation, la localisation de ses vergers, ses pratiques culturales, etc.).

\section{Des producteurs maîtres de leurs choix techniques dans le processus de production}

Dans ce modèle, l'OP ne cherche pas à concentrer la production, et il n'y a donc ni préconisations variétales, ni cahier des charges collectif, ni appui technique contrôlé par l'OP. Chaque producteur conserve sa propre stratégie productive et commerciale (Dubois de Montmarin, 2003), certains visant le haut de gamme et d'autres les bas coûts de production. C'est le contact direct avec les metteurs en marché (de façon informelle ou par cahier des charges interposé) et le niveau de rémunération qui sont les principaux moteurs du changement des pratiques.

\section{L'OP comme structure organisationnelle d'ajustement}

À l'opposé du modèle précédent, ce modèle d'OP cherche à relier très étroitement les opérations de production et de commercialisation, en responsabilisant le producteur autour d'engagements qui ne doivent pas concerner seulement une production mais aussi une commercialisation. Ce modèle se traduit par une organisation assez légère. L'OP peut ainsi n'être qu'un bureau d'enregistrement des opérations de facturation et l'interlocuteur des pouvoirs publics pour la mise en œuvre du programme opérationnel. Mais elle peut aussi développer une compétence marchande propre, en recensant et traitant les informations sur les différents actes de vente de ses adhérents, pour pouvoir identifier les circuits les plus rémunérateurs pour une qualité donnée. Une telle organisation suppose une forte capacité d'apprentissage à travers le retour d'expérience. L'instabilité inhérente à ces relations, qui par principe ne peuvent faire reposer la confiance sur leur pérennité, doit alors être composée par l'adaptation très particulière de l'offre fournie à la réalité du circuit. Ce système a cependant une limite, qui est la difficulté, voire l'impossibilité, à 
atteindre la grande distribution. En effet, il est impossible d'obtenir de gros volumes de qualité homogène au niveau d'une seule exploitation, ou même au niveau du collectif, du fait de la diversité des exploitations.

\section{Le modèle club : un club de producteurs maîtrisant la commercialisation}

Le modèle club est une forme organisationnelle intermédiaire qui permet à un groupe de producteurs associés avec des objectifs très précis de qualité au verger et qui valorisent cette qualité dans des circuits commerciaux adaptés.

\section{Une orientation préférentielle sur les marchés haut de gamme}

Ce modèle d'OP vise un positionnement commercial de type niche, qui leur permet de valoriser une qualité haut de gamme envisagée dès la plantation du verger. Les clients sont alors des opérateurs économiques qui visent eux-mêmes un positionnement qualitatif haut de gamme: exportateurs opérant dans des régions permettant la valorisation de ces niveaux qualitatifs (comme l'Angleterre ou la Suisse, par exemple), distributeurs français positionnés sur des niches de clientèles haut de gamme (par exemple certaines enseignes urbaines de proximité). L'activité commerciale repose sur des investissements de forme réalisés hors saison qui permettent la prospection de clients très spécifiques, sélectionnés et démarchés en fonction des stratégies de niche qu'ils déploient et la mise en place de partenariats qui permettent de spécifier des produits (taux de sucre garantis, degrés de maturité mesurés) ou des services (emballages spécifiques, conditions de transports spécifiant les écarts de température tolérés). Les producteurs s'investissent euxmêmes dans la construction de partenariats avec les clients, par exemple en recevant certains clients dans leurs exploitations pour leur montrer certaines contraintes techniques et les spécificités de la production. Le prin- cipe est de valoriser au mieux commercialement le positionnement qualitatif haut de gamme à la fois des produits mais également des exploitations, souvent en pointe dans le domaine environnemental (production fruitière intégrée), mais aussi organisationnel (HACCP ${ }^{11}$, Norme ISO 14001). Ces stratégies d'apprentissage permettent de conserver une flexibilité, notamment face à des opérateurs comme la GMS qui cherchent à banaliser les éventuels gains qualitatifs obtenus, en demandant aux producteurs de baisser les coûts une fois qu'ils considèrent le produit suffisamment stabilisé. Il s'agit donc d'une démarche d'innovation permanente destinée à maintenir dans un équilibre relatif et temporaire le rapport de force qui se joue entre les producteurs et la grande distribution. C'est dans ce contexte qu'une stratégie de cueille à maturité sur l'arbre, reposant sur une logistique particulière entre les producteurs et les clients, a pu être envisagée. Pendant la campagne, la commercialisation est gérée par une société de commercialisation qui permet de décharger les producteurs des contraintes de la mise en marché. La société de commercialisation s'occupe des commandes mais déclenche aussi certaines opérations de production (la cueille et le conditionnement au verger) en flux tendu. Le produit est vendu au jour j par les commerciaux de l'entreprise, puis ramassé et emballé à j+1 par l'exploitant pour être livré le même jour chez le client.

\section{Un accord tacite entre gros producteurs sur les qualités à produire}

Ce modèle d'OP correspond à l'agrégation d'exploitations de superficies généralement supérieures à la moyenne et qui ont des stratégies comparables de qualité haut de gamme. Chaque producteur est individuel-

11. La méthode (HACCP) Hazard Analysis Critical Control Points a été développée dans l'industrie agroalimentaire pour contrôler la sécurité sanitaire des aliments à partir d'un certain nombre de points critiques du processus de fabrication. 
lement trop petit pour exister sur le marché et trop gros pour aller se fondre dans une structure coopérative, jugée trop hétérogène. L'entrée des nouveaux producteurs se fait par cooptation, sur les valeurs qui fondent le collectif, dans un objectif d'augmentation des tonnages et d'étalement de la production (d'où un élargissement progressif du bassin d'approvisionnement).

Dans ce modèle d'OP, avec très en amont un accord sur les choix de production lors de la sélection des exploitations et en aval une bonne valorisation de la qualité supérieure, il n'est pas nécessaire de contraindre très fortement les décisions de culture des producteurs: il existe une forte homogénéité des méthodes de travail autour d'un référentiel technique exigeant, décidé en commun, mais non imposé. Au technicien « coordonnateur» et polyvalent de l'OP hiérarchique fait donc place un technicien parfois externe à la structure, dont les producteurs attendent des conseils très pointus sur les techniques jugées cruciales dans l'élaboration de la qualité (et en particulier la protection phytosanitaire et le déclenchement des récoltes). De plus, ce modèle cherche à faciliter l'adaptation des exploitations aux nouvelles exigences de la filière, par exemple en élaborant un tableau de bord qui récapitule l'ensemble des exigences des différents clients, auquel la totalité des producteurs doit se soumettre (cahiers des charges des différentes GMS, EurepGap...), ou encore en donnant à l'exploitation-mère à l'origine de la structure un rôle de vitrine technique dont les autres exploitations peuvent s'inspirer.

\section{L'OP comme créateur de marchés qualitatifs}

La structure organisationnelle de l'OP est ici assez légère, dans la mesure où le plus gros de la coordination avec les producteurs se situe très en amont, c'est-à-dire dans la possibilité même de faire partie du club, d'où le principe de cooptation pour l'entrée. En cours de production, plusieurs éléments concourent à un auto-ajustement de chaque producteur avec la structure collective. D'une part, le producteur est informé en temps réel du prix de vente de sa propre production, et peut analyser les conséquences économiques de ses choix techniques. D'autre part, contrairement à l'OP hiérarchique où l'étalement de la production est un enjeu fort de coordination avec les producteurs, dans ce cas, chaque exploitation cherche par elle-même à étaler la production pour des questions d'organisation du travail, à cause de sa taille. Enfin, la plupart des exploitations adhérentes a une surface suffisante pour atteindre individuellement le segment des GMS, et donc élaborer ses propres lots de fruits sans les combiner à ceux d'autres exploitations, réduisant le besoin d'homogénéiser les choix variétaux par exemple.

Cependant, la structure collective joue un rôle important de surveillance, pour éviter les dérives éventuelles des individus qui pourraient mettre à mal l'image « haut de gamme » de l'OP. Ainsi en est-il du rôle du responsable qualité, chargé de coordonner l'agréage dans les différentes stations de conditionnement des producteurs : en effet, une dérive dans les critères d'agréage dans une station, en particulier sur les critères de qualité non objectivables par des mesures, serait préjudiciable à l'ensemble de la structure puisque les différents producteurs ont en commun plusieurs clients.

L'OP apparaît ici comme l'opérateur principal d'une qualité qui se définit dans le cadre même des positionnements techniques des producteurs. Les marchés qu'ils visent, d'une certaine manière, ne préexistent pas à leur action, mais sont véritablement le produit des ajustements que l'OP est en mesure de construire entre des objectifs techniques de production et des opportunités de commercialisation qu'ils suggèrent à leurs clients.

Cette grille analytique permet de décrire 
la réalité du paysage rhône-alpin, au sens où les différentes OP qui s'y trouvent se rapprochent de chacun de ces idéaux-types. Sur les 17 OP, commercialisant de la pêche et/ou de l'abricot en Rhône-Alpes, plus de la moitié (9 sur 17) se rattache au modèle hiérarchique avec délégation ; à l'opposé dans 3 OP, les producteurs négocient directement avec les opérateurs de la filière ; 3 OP combinent les deux formules, offrant ainsi à leurs adhérents la possibilité de prendre en charge l'acte commercial ou au contraire de le déléguer à $1^{\prime} \mathrm{OP}^{12}$ et $2 \mathrm{OP}$ se rattachent au modèle club. A priori la convergence avec un modèle du type club ne peut pas être repérée dans les statistiques, même si nos enquêtes montrent aux moins l'existence de deux exemples de structures s'en rapprochant, l'une avec délégation de la fonction commerciale à une structure externe, l'autre avec une gestion en direct et centralisée de cette fonction. Malgré tout, ces modèles ne sont ni statiques, ni exclusifs dans leurs modalités organisationnelles. Par exemple, même dans les OP de type hiérarchique ou club, caractérisées par un accord collectif sur les objectifs de production et/ou les méthodes, le tri à la récolte est un point de passage obligé pour limiter la variabilité inhérente à la dimension biologique de la production. Inversement, les OP de type fédératif, qui se veulent assez souples dans leurs modes de coordination voient les limites de leur système dans la difficulté à atteindre le créneau des GMS faute d'un minimum de concertation. Enfin, comme nous l'avons indiqué plus haut, il faut ajouter que de nombreux producteurs/expéditeurs se

12. Cette distinction statistique, réalisée à partir des statistiques du BRM, déjà difficile aujourd'hui, ne sera plus possible dans l'avenir puisque officiellement la délégation deviendra obligatoire, même si les pratiques sociales de négociation en direct producteurs/expéditeurs ou grossistes, au dire des acteurs concernés, perdureront et seront entérinées par les OP. situent hors $\mathrm{OP}^{13}$. Nous faisons l'hypothèse qu'ils cherchent ainsi à échapper à des formes de coordination portant sur les choix productifs ou les choix de mise en marché qu'ils jugent trop contraignantes dans un contexte où ils estiment pouvoir tirer une rente de circuits commerciaux dans lesquels la confiance interpersonnelle reste encore dominante. Nous n'avons pas eu l'occasion de creuser cet aspect dans notre enquête, mais il semble nécessaire de mieux comprendre les ressorts de cette stratégie de producteurs, car les profils de ceux qui quittent les OP ou refusent d'y entrer ne sont aucunement homogènes ni en taille d'exploitation, ni en stratégie de qualité. Il semble que l'on trouve ici une conséquence directe de la nouvelle OCM.

Il pourrait être intéressant d'utiliser cette grille d'analyse pour caractériser les OP des autres bassins producteurs en France et en Europe et notamment de ceux qui concurrencent fortement la production rhône-alpine (comme par exemple le Gard ou la région de Murcia).

\section{Discussion de la planification à la flexibilité}

Nous voudrions ici présenter les trois modèles sous une autre perspective, celle du type d'organisation industrielle qu'ils mettent en œuvre. Cette optique permet notamment de mieux comprendre la nature des relations que les OP cherchent à construire avec leurs clients mais aussi le rapport de dépendance qui s'instaure avec leurs partenaires amont ou aval (Thoenig et Dupuy, 1989), elle met en évidence la contribution des outils et des stratégies de gestion aux formes d'interdépendances entre les acteurs du marché.

13. On peut estimer en 2004 approximativement à 30 \% en Rhône-Alpes la part du volume en pêches et abricots qui est commercialisée hors OP ; si on raisonnait en nombre de producteurs, le pourcentage serait plus élevé, de l'ordre de $50 \%$ environ. 
Les trois idéaux-types que nous avons définis combinent en effet, des règles renvoyant à deux formes différentes de planification industrielle et de flexibilité (Soler $e t$ $a l, 1995)$. La première permet de traiter des quantités importantes en anticipant au maximum les aléas qui peuvent survenir en cours de production et de commercialisation. La seconde est mobilisée pour rechercher le meilleur prix à un moment donné, la flexibilité permettant au maximum l'adaptation aux conditions du moment. Sortir de ce dilemme conduit forcément à des compromis, que les trois idéaux types vont réaliser dans des conditions différentes.

- Les OP se référant au modèle hiérarchique, gérant souvent des volumes plus importants que les autres (avec un nombre élevé de producteurs) vont chercher à sécuriser au maximum leurs relations commerciales et les relations avec les producteurs, en étant exigeantes par rapport à ceux-ci sur la qualité des marchandises apportées, les espèces et variétés recherchées. Ces OP ont souvent un statut de type coopératif, mais pas exclusivement, qui garantit la fidélité à la structure. L'efficacité de ces structures réside dans leur capacité à gérer les producteurs en fonction des marchés qu'elles ont réussi à stabiliser. Pratiquement on trouve dans cette situation les bons élèves de la réforme de l'OCM, qui ont su combiner dynamisme commercial et planification de la production. Elles tendent à s'écarter graduellement du modèle coopératif pour s'apparenter de plus en plus au modèle de la grande entreprise. Elles cherchent à contrecarrer le pouvoir grandissant de la grande distribution en jouant sur le même terrain, c'est-à-dire celui de la taille, c'est la raison pour laquelle elles ne sont pas loin d'envisager des alliances entre elles. De telles associations permettraient de sécuriser à la fois des apports réguliers, mais aussi des circuits. Dans les OP se référant à un modèle hiérarchique, la flexibilité rendue nécessaire par les aléas climatiques et marchands est reportée sur les producteurs, à charge pour eux d'accélérer la récolte ou de trier avec plus ou moins de rigueur en fonction de l'état du marché. Cependant, un autre moyen de plus en plus fréquent de répondre à cette nécessité de gérer des aléas est de créer des filiales commerciales qui, par une politique d'achats extérieurs à l'OP, vont externaliser les inconvénients de cette nécessité de flexibilité.

- Les OP se référant au modèle fédératif vont en général traiter globalement des volumes de fruits moins importants et elles sont souvent constituées de producteurs plus hétérogènes, à la fois par la taille et par l'activité, puisque certains sont eux- mêmes aussi des metteurs en marché ; la planification de la mise en marché est de fait réalisée par chacun des producteurs qui reste très fidèle au metteur en marché à qui il livre sa marchandise ; en revanche dans cette relation marchande suivie, le metteur en marché qui lui aussi tient à garder le producteur, s'engage à valoriser au mieux toute les qualités commerciales produites, réalisant des ajustements, en fonction de son propre réseau commercial, construit en tenant compte aussi de cette finalité. L'OP va ajouter ici une flexibilité supplémentaire comme opérateur permettant d'optimiser le choix d'un metteur en marché en fonction de sa capacité à rémunérer telle ou telle qualité dans ses circuits.

- Les OP qui se réfèrent à un modèle club, ressemblent plus au modèle hiérarchique qu'au modèle fédératif; il y a planification de la production dans la construction même d'un petit groupe de producteurs choisissant de travailler ensemble parce qu'ils sont complémentaires pour gérer des marchés de qualité supérieure, avec une grande rigueur technique. Le petit nombre de producteurs, la place de la production de chacun dans l'ensemble ne permet pas d'ajus- 
tements importants, par rapport à des marchés où l'OP profite d'une réputation d'entreprise à laquelle elle ne doit pas faillir ; la flexibilité par rapport aux aléas ne va jouer qu'en interne entre producteurs se faisant confiance, en s'appuyant sur le caractère très fortement interpersonnel de la construction.

Enfin, le quatrième modèle correspond à une faible coordination des producteurs et une faible valorisation de la variabilité qualitative. Il pourrait recouvrir le cas des coopératives en perte de vitesse dans la région, qui voient progressivement leurs adhérents quitter la structure parce qu'elle ne parvient pas à se doter d'une organisation qui permettrait une bonne valorisation de la qualité produite et qu'elle se contente de déléguer cette tâche à leurs clients.

\section{Conclusion}

A travers cette diversité de façons qu'ont les OP d'organiser des pratiques productives en amont et de travailler le marché en aval, nous avons voulu souligner l'action stratégique de cet acteur essentiel du paysage agricole régional en matière de construction de la qualité. Si ces actions découlent directement de contraintes et d'opportunités dessinées par la nouvelle OCM, elles traduisent également la capacité des acteurs à s'organiser pour opérer de manière spécifique sur le marché des fruits d'été, à travers des stratégies de valorisation des variabilités qualitatives très différenciées.

On peut s'interroger sur les raisons de la coexistence de ces différentes formes d'OP, et plus particulièrement ce qui explique que les avantages liés à une organisation fortement anticipée en termes de positionnement commercial ne l'ont pas définitivement emporté sur la préoccupation de flexibilité.
Il est probable que le caractère périssable des fruits frais qui nécessite de garder une grande flexibilité pour gérer les aléas momentanés (décalage de récolte lié à une canicule, perte de qualité liée à des intempéries) explique certainement cette réalité. Cette relation entre la nature périssable du produit et la forme de l'OP n'est pas traitée pour le moment par la littérature et il semble bien qu'il pourrait être intéressant d'élargir la perspective dans de futures recherches, notamment en mettant en regard les formes d'OP avec les types de fruits (fruits stockables comme les pommes et non stockables comme les pêches). Mais cette variable ne semble pas expliquer l'ensemble de l'hétérogénéité des formes d'OP et il est nécessaire de regarder la façon dont elles envisagent leurs relations au marché. Dans le même sens, la production de pêches en France n'est pas une production excédentaire (importation de $20 \%$ des tonnages), qui nécessiterait une politique d'exportation très active ; il s'agit plus d'ajustement par rapport au marché national que de conquête d'un marché extérieur (en l'occurrence l'Allemagne), ou les exportateurs de pays du Sud bénéficient d'avantages comparatifs considérables (calendrier, taux de sucre, prix de revient). Il en serait tout autrement pour les pommes où la capacité de production française, (deux fois la consommation nationale) et les possibilités de stockage, obligent et permettent de piloter la commercialisation, avec un poids plus grand donné à la planification de la commercialisation des différentes qualités sur toute l'année et avec la nécessité de trouver une place croissante sur les marchés extérieurs (Lacroix et al., 2004). Cette situation nous conduit à ne pas négliger les propriétés spécifiques des produits dans l'explication des formes mêmes des relations marchandes (Vatin, 1995). 


\section{RÉFÉRENCES BIBLIOGRAPHIQUES}

Akerlof G.A. (1970). The market for "lemons": quality, uncertainty and the market mechanism. The Quarterly Journal of Economics, 84, p. 488-500.

Aulagnier M. (1996). Potentialité régionales et démarches qualité, le cas de la pêche de la Drôme. Ingénieries, EAT, $\mathrm{n}^{\circ} 7$.

Barrey S. (2004). Le travail marchand dans la grande distribution alimentaire. La définition des relations marchandes, Thèse de sociologie de l'Université de Toulouse II.

Cochoy F., Dubuisson-Quellier S. (2000). Les professionnels du marché : vers une sociologie du travail marchand, Sociologie du travail, $\mathrm{n}^{\circ} 3$, Vol. 42.

Codron J.-M., Giraud-Héraud E., Soler L.-G. (2003). French large-scale retailers and new supply segmentation strategies for fresh products. Washington (USA), Workshop "Global markets for highvalue foods", 14/02/2003, $14 \mathrm{p}$.

Dubois de Montmarin B. (2003). Les déterminants économiques des choix de production dans un échantillon d'exploitations fruitières de la Drôme. Mémoire de fin d'études ENESAD / INRA UMR Innovation, 83 p. + annexes (dir. J. Pluvinage).

Dubuisson-Quellier S., 2003-a. Goût des produits et goûts des consommateurs. La pluralité des épreuves de qualification dans la mise en marché des produits alimentaires. In Dubuisson-Quellier et Neuville (Dir.), «Juger pour échanger », Inra Editions, MSH, p. 47-74.

Dubuisson-Quellier S. (2003-b). Confiance et qualité des produits alimentaires : une approche par la sociologie des relations marchandes, Sociologie du travail, $\mathrm{n}^{\circ} 1$.

Eymard-Duvernay F. (1989). Conventions de qualité et formes de coordination. Revue économique (2), p. 329-359.
Friedberg E. (1993). Le pouvoir et la règle. Dynamique de l'action organisée, Paris, Seuil, 405 p.

Giauque P., Moras P., Moreau-Rio M.-A., Scandella D., Kraeutler E. (1997). La pêche, consommation et itinéraire qualité. Ed CTIFL, 96 p.

Hassan D., Simioni M. (2001). Filière fruits et légumes: comment la grande distribution transmet-elle aux consommateurs les variations de prix à la production? INRA Sciences Sociales, Recherches en économie et sociologie rurale, $\mathrm{N}^{\circ} 4 / 00$.

Karpik L. (1989). L'économie de la qualité. Revue française de sociologie, $\mathrm{n}^{\circ} 30$, p. 187-210.

Lacroix P., Jacquet F., Pluvinage J. (2004). Les orientations de la production de fruits à pépins dans un contexte de production fruitière intégrée. Illustration dans le SudEst de la France à partir des stratégies de différentes organisations de producteurs et de leurs adhérents, Montpellier, INRAUMR Innovation/IAMM, $130 \mathrm{p}$.

Le Bail M. (2005). Le bassin d'approvisionnement : territoire de la gestion agronomique de la qualité des productions végétales. In «Agronomes et territoires », deuxièmes entretiens du Pradel, Paris, Ed. l'Harmattan, 502 p.

Montigaud J.-C., Rio P., Martinez R. (2002). L'OCM fruits et légumes dans le Sud-Est de la France : une tentative de bilan. Série Etudes, Montpellier, Ed. INRA-UMR Moïsa, $\mathrm{n}^{\circ} 2,66 \mathrm{p}$.

Navarrete M., Tordjman S., Rouby A. (2003). La planification des plantations par les structures de première mise en marché dans la filière fruits et légumes. Comparaison des cas de la salade et de la pêche dans le Sud-Est de la France. Fruits, $\mathrm{n}^{\circ}$ 58, p. 261-74.

Navarrete M., Le Bail M., Papy F., Bressoud F., Tordjman S. (2006). Combining lee- 
way on farm and supply basin scales to promote technical innovations. In «Lettuce production». Agronomy for Sustainable Development, $\mathrm{n}^{\circ}$ 26, p. 77-87. Puypalat J.-E. (2002). Configurations de mise en marché. Le cas de la pêche en Rhône-Alpes. Mémoire pour le DEA de Sociologie de l'Institut d'Etudes Politiques de Paris / INRA-SAD, Unité d'Ecodéveloppement Avignon, 102 p. + annexes (dir. Ch. de Sainte Marie et S. Dubuisson- Quellier).

Pluvinage J. (2004). Les Organisations de Producteurs, instrument clef de l'intervention publique communautaire et nationale, dans le secteur des fruits et légumes. Campagnes solidaires.

Pluvinage J., de Sainte-Marie C., Bellon S., Chazoule C., Dubuisson-Quellier S., Fauriel J., Navarrete M., Plenet D. (2005). Valoriser par la qualité les fruits frais en Rhône-Alpes : passer d'une incantation à la mise en œuvre d'une stratégie régionale ? Lyon, Symposium PSDR, 9-11 mars, Communication orale et rapport scientifique, $21 \mathrm{p}$.

Rouby A. (2001). Les choix de plantation des producteurs de pommes et de pèches dans le Bassin Rhône-Méditerrannée : facteurs pris en compte dans les vergers et influences d'opérateurs extérieurs. Mémoire DAA Agronomie-Environnement, INAPG, 64 p. + annexes. (dir. M. Navarrete).

Sainte-Marie (de) C., Valceschini E. (1994). La qualité des produits : une construction sociale. Études et Recherches sur les systèmes agraires et le développement, $\mathrm{n}^{\circ} 28$, p. 75-79.

Sainte-Marie (de) C., Agostini D. (2003). Du signe à l'inscription géographique de l'origine : la requalification de la clémentine de Corse. In «Juger et échanger ». S. Dubuisson-Quellier et J.-P. Neuville éds., Paris, INRA-MSH, p. 185-212. Simon M. (2003). La segmentation de l'offre en fruits dans la grande distribution de Rhône-Alpes. Proposition de positionnements pour la production régionale. Mémoire de DAA Agronomie - Environnement, INAPG/ INRA ESR, 52 p. + annexes (dir. L.-G. Soler).

Soler L.-G., Tanguy H., Valceschini E. (1995). Problèmes de planification, systèmes de gestion et organisation interne de la firme. Cahiers d'économie et sociologie rurales, $\mathrm{n}^{\circ} 37$, p. 202-225.

Thoenig J.-C. et F. Dupuy. (1989). La loi du marché, Paris, L'Harmattan.

Trompette P. (2005). Secteur funéraire : une économie de la captation. Revue Française de sociologie, V.46, $\mathrm{n}^{\circ}$ 2, p. 233-264.

Vatin F. (1995). Le lait ou la raison marchande, Rennes, PUR.

Vidal M. (2003). Comment se coordonnent les stratégies d'approvisionnement des Organisations de Producteurs et les stratégies de production des exploitants agricoles? Le cas de la production de pêche en Rhône-Alpes. Mémoire DAA Agronomie-Environnement, INAPG, 56 p. + annexes (dir. M. Navarrete). 